Pacific Journal of Mathematics

QUOTIENTS OF NEST ALGEBRAS WITH TRIVIAL 


\section{QUOTIENTS OF NEST ALGEBRAS WITH TRIVIAL COMMUTATOR}

\section{GARETH J. KNOWLES}

The main result of this paper is to show that every operator $T$ commuting with a nest algebra modulo a two-sided ideal $\mathscr{J}$ of $\mathscr{L}(H)$ is of the form $T=\lambda I+J$ for some $\lambda \in C, J \in \mathscr{J}$.

Introduction. The structure of commutators of non-selfadjoint operator algebras has received considerable interest in recent years $[4,5,6,8$, 9, 13, 16 and their references] ([7] contains a good survey of known results). However, results for perturbed algebras in general and finite perturbations in particular are not available except for the special case of the ideal $\mathscr{K}$ of all compact operators. To put the results proven here into perspective, we mention two well known and particularly useful special cases. For any subalgebra $\mathscr{A}$ of $\mathscr{L}(H)$ and any subset $\mathscr{M}$ of $\mathscr{L}(H)$, denote by $C(\mathscr{A}, \mathscr{M})$ the collection $\{T \in \mathscr{L}(H): A T-T A \in \mathscr{M}$ for every $A \in \mathscr{A}\}$. We now state:

I. (Calkin [3].) Given any ideal $\mathscr{J}$ (two-sided) of $\mathscr{L}(H)$,

$$
C(\mathscr{L}(H), \mathscr{J})=C I+\mathscr{J} .
$$

Using the results of Johnson and Parrott [11] on $C(\mathscr{B}, \mathscr{K})$ for $\mathscr{B}$, a type I von Neumann algebra, Christensen and Peligrad were able to show the following.

II. (Christensen and Peligrad [5].) For any nest algebra $\mathscr{A}$,

$$
C(\mathscr{A}, \mathscr{K})=C I+\mathscr{K} \text {. }
$$

It should be mentioned that II was shown to have an extension to the von Neumann-Schatten $p$-classes in [7].

The central result of this paper is to show that I and II above are "endpoints" of a very general theorem concerning commutators. This result can be stated as:

III. For any nest algebra $\mathscr{A}$ and any ideal $\mathscr{J}$ of $\mathscr{L}(H)$,

$$
C(\mathscr{A}, \mathscr{J})=C I+\mathscr{J} \text {. }
$$


Combining III with the main result of [4], we obtain:

IV. Any derivative of a nest algebra into an ideal (two-sided) $\mathscr{J}$ of $\mathscr{L}(H)$ is implemented by an operator from $\mathscr{J}$.

I would like to thank C. Apostol for his helpful conversation.

For the purpose of this paper, $\mathscr{A}$ will denote the nest algebra of all operators acting on a fixed separable Hilbert space $H$ leaving invariant a (complete) totally ordered nest of subspaces $N$. Denote by $\mathscr{E}$ the corresponding totally ordered nest of orthogonal projections onto the members of $\mathscr{N}$. If $\mathscr{E}=\left\{E_{n}\right\}_{n \in Z}$, let $\Delta_{i}$ be the orthogonal projection $E_{i}-E_{i-1} \cdot \mathscr{J}$ will denote an arbitrary but non-zero two-sided ideal of $\mathscr{L}(H)$. It is well known [10] that $\mathscr{F} \subseteq \mathscr{J} \subseteq \mathscr{K}$, where $\mathscr{F}$ denotes the ideal of all finite rank operators. (Note that all the results below are obviously true for $\mathscr{J}=(0)$.)

Essential use will be made of the identification between such an ideal $\mathscr{J}$ and its corresponding ideal set $\tilde{\mathscr{J}}$ of $s$-numbers in $c_{0}(N)$ satisfying

(i) $\left\{\lambda_{i}\right\},\left\{\mu_{t}\right\}$ in $\tilde{\mathscr{J}}$ implies $\left\{\lambda_{t}+\mu_{t}\right\}$ in $\tilde{\mathscr{J}}$.

(ii) $\left\{\lambda_{l}\right\} \in \tilde{\mathscr{J}}$ and $0 \leq \mu_{i} \leq \lambda_{i}$ for every $i \in N$ implies $\left\{\mu_{t}\right\} \in \tilde{\mathscr{J}}$. in $\tilde{\mathscr{J}}$.

(iii) For any permutation $\pi: \mathbf{N} \rightarrow \mathbf{N},\left\{\lambda_{i}\right\}$ in $\tilde{\mathscr{J}}$ implies that $\left\{\lambda_{\pi(i)}\right.$ is

This identification is given by $s: T \rightarrow \sigma\left(\left(T^{*} T\right)^{1 / 2}\right)$. We will use the standard notation $s_{j}(T)$ for the $j$ th eigenvalue of $\left(T^{*} T\right)^{1 / 2}$. Given $T$ in $\mathscr{L}(H)$, denote by $\delta_{T}$ the map from $\mathscr{A}$ to $\mathscr{L}(H)$ given by $\delta_{T}(A)=A T-$ $T A$. Let $x \otimes y$ be the rank one operator $(x \otimes y) z=\langle z, x\rangle y$. By c.l.s. $\{S\}$ will be meant the closed linear span in the norm topology of the set $S$.

Commutants of nest algebras modulo $\mathscr{J}$. In order to prove III, we initially divide the problem into three cases:

(i) There exists a projection $E$ into $\mathscr{E}$ with infinite range and kernel.

(ii) There exists an increasing sequence $\left\{E_{n}\right\}_{n=0}^{\infty}$ of finite dimensional projections in $\mathscr{E}$, with $E=\sup E_{n}$ having finite dimensional kernel.

(iii) There exists a decreasing sequence $\left\{E_{n}\right\}_{n=0}^{\infty}$ of finite co-dimensional projections in $\mathscr{E}$, with $E=\inf E_{n}$ having finite range.

Case (i). As in [5] we note that there will exist a partial isometry $V$ in $\mathscr{A}$ with $V V^{*}=E$ and $V^{*} V=I-E$. Thus both $E \mathscr{L}(H) E V$ and $V(I-E) \mathscr{L}(H)(I-E)$ are subsets of $\mathscr{A}$. Let $\delta_{K}$ be a (bounded) derivation from $\mathscr{A}$ into $\mathscr{J}$. For any $X$ in $\mathscr{L}(H), \delta_{K}(E X E V)=$ $\delta_{K}(E X E) V+E X E \delta_{K}(V)$, it will immediately follow that $\delta_{k}(E X E) E$ is 
in $\mathscr{J}$. Define the ideal $\mathscr{J}_{1}$ of $\mathscr{L}(E H)$ to be

$$
\mathscr{J}_{1}=\{E T E: T \in \mathscr{J}\} \text {. }
$$

Consider the action of $\delta_{E K E}$ on $\mathscr{L}(E H)$. For any $X$ in $\mathscr{L}(H)$,

$$
\delta_{E K E}(E X E)=E(X E K-K E X) E=E \delta_{K}(E X E) E .
$$

Thus $\delta_{E K E}$ derives $\mathscr{L}(E H)$ into $\mathscr{J}_{1}$. An application of I above will show that $E K E=\lambda E+T_{1}$ for some $T_{1}$ in $\mathscr{J}_{1}$. An exactly similar argument will show that $(I-E) K(I-E)$ is of the form $\mu(I-E)+T_{2}$, where $T_{2}=(I-E) T_{2}(I-E)$ for some $T_{2} \in \mathscr{J}$. In addition, $E K(I-E)=$ $E \delta_{K}(E)(I-E)=E T_{3}(I-E)$ with $T_{3} \in \mathscr{J}$. Similarly, $(I-E) K E=$ $(I-E) \delta_{K}(I-E) E=(I-E) E T_{4} E$ with $T_{4} \in \mathscr{J}$. Therefore, $K$ can be written as:

$$
K=\left[\begin{array}{ll}
\lambda & \\
& \mu
\end{array}\right]+\left[\begin{array}{ll}
T_{1} & T_{4} \\
T_{3} & T_{2}
\end{array}\right],
$$

where the second term $T$ is in $\mathscr{J}$. All that remains is to show $\lambda=\mu$. Note, however, that since $V \in \mathscr{A}$, we have

$$
(\lambda E+\mu(I-E)+T) V-V(\lambda E+\mu(I-E)+T) \in \mathscr{J} .
$$

It immediately follows that $(\lambda-\mu) E \in \mathscr{J}$, showing $\lambda=\mu$.

Case (ii). In order to prove case (ii), it will be necessary to further subdivide case (ii) into (ii) (a) $\mathscr{J} \neq \mathscr{F}$ and (ii) (b) $\mathscr{J}=\mathscr{F}$. Before beginning the proof of either, we note that it may as well be assumed that $\mathscr{E}$ is the classical nest of one-dimensional jumps on $l^{2}(N)$. That is, with respect to the usual basis $\left\{e_{j}\right\}_{n=1}^{\infty}, E_{n}$ is given as the projection onto the closed linear span of $\left\{e_{j}\right\}_{j=1}^{n}$.

Case (ii)a. Let $\delta_{K} \cdot \operatorname{Alg}\left\{E_{n}\right\} \rightarrow \mathscr{J}$. It follows from II that we can assume $K$ is compact. Fix a $c_{0}(N)$ sequence $\left\{\varepsilon_{i}\right\}$ in $\tilde{\mathscr{J}}$ satisfying $\varepsilon_{1}>\varepsilon_{2}>\cdots>0$. Define a partial isometry $A$ in $\mathscr{A}$ by $A^{*} e_{i}=e_{n_{i}}$, where $n_{i}>n_{i-1}$ and $\left\|\Delta_{n_{i}} A K\right\|<2^{-i} \varepsilon_{i}$. That this is possible follows from the compactness of $K$ and the observation that $\left(I-E_{n}\right) \downarrow 0$ strongly. It can now be seen that $A K$ is the operator with the property that $\Delta_{n} A K=$ $\Delta_{n_{i}} K$. We claim that $s(A K)$ is dominated by $\left\{\varepsilon_{i}\right\}$, and thus $A K \in \mathscr{J}$ by (iii). That this holds is an application of [1]. Indeed we have

$$
s_{n+1}(A K) \leq\left\|\left(I-E_{n}\right) A K\right\| \leq \sum_{j=n+1}^{\infty}\left\|\Delta_{j} A K\right\|<\varepsilon_{n+1}
$$

since, in particular, rank $E_{n} A K \leq n$. 
Thus, necessarily $K A$ is also in $\mathscr{J}$. Moreover,

$$
s(K A)=s\left(A^{*} K^{*}\right)=\sigma\left[\left(K A A^{*} K^{*}\right)^{1 / 2}\right]=\sigma\left[\left(K K^{*}\right)^{1 / 2}\right]=s(K),
$$

showing $K$ is also in $\mathscr{J}$.

Case (ii)b. It is not too difficult to show that this result follows from case (ii)a using the fact that $\cap\{\mathscr{J}: \mathscr{J} \supsetneqq \mathscr{F}\}=\mathscr{F}$. However, the following proof is of independent interest in that it provides a concrete example of an operator $A$ such that $\left\{\delta_{T}(A) \notin \mathscr{F}\right.$ for a given $T \notin C I+\mathscr{F}$. Since $\delta_{X}(A) \subseteq \mathscr{F}$ if and only if $\delta_{X^{*}}\left(A^{*}\right) \subseteq \mathscr{F}$, it may as well be assumed that $\mathscr{A}$ is the algebra of all (bounded) lower triangular matrices with respect to the basis $\left\{e_{n}\right\}$. Let $\delta_{T}: \mathscr{A} \rightarrow \mathscr{F}$. Suppose, contrary to the assertion of III, that $T \notin C I+\mathscr{F}$. We shall construct sequences $\left\{x_{n}\right\},\left\{y_{n}\right\}$ of unit vectors together with associated projections $E_{m(n)}$ and $E_{j(n)}$ satisfying

(i) $\left\langle x_{j}, x_{k}\right\rangle=\left\langle T x_{j}, x_{k}\right\rangle=0$ for $j \neq k$.

(ii) $x_{n}=E_{m(n)} x_{n}$ and $y_{n}=\left(E_{j(n)}-E_{m(n)}\right) y_{n}$.

(iii) $\left\{T y_{k}-\left\langle T x_{k}, x\right\rangle y_{k}\right\}_{k=1}^{n}$ are linearly independent vectors for each $n \in N$. The construction is an inductive one.

$k=1$. Let $x_{1}=e_{1}$. If for every $e_{j}, j>1, T e_{j}=\left\langle T e_{1}, e_{1}\right\rangle e_{j}$, it will immediately follow that $T=\left\langle T e_{1}, e_{1}\right\rangle I+K$ for $K$, a rank two operator, contrary to our assumption. Take $y_{1}=e_{k}$, where $k$ is the first integer with $T e_{k} \neq\left\langle T e_{1}, e_{1}\right\rangle e_{k}$. It is easily seen that $\left(x_{1}, y_{1}\right)$ satisfies (i), (ii) and (iii) above.

$k=n$ implies $k=n+1$. Suppose that $\left\{x_{i}\right\}_{i=1}^{n}$ and $\left\{y_{i}\right\}_{i=1}^{n}$ have been chosen to satisfy (i) through (iii). Let $H_{n}$ be c.l.s. $\left\{x_{1}, \ldots, x_{n}\right.$, $\left.T x_{1}^{*}, \ldots, T x_{n}^{*}\right\}$ and note that $E_{2 n+1}\left(H_{n}\right) \varsubsetneqq E_{2 n+1}(H)$. From this we deduce the existence of a unit vector $x_{n+1}=E_{2 n+1} x_{n+1}$ satisfying (i) for $j, k \leq n+1$. Take $E_{m(n+1)}=E_{2 n+1}$.

Define $\tilde{H}_{n}$ to be c.l.s. $\left\{y_{1}, \ldots, y_{n}, T y_{1}^{*}, \ldots, T y_{n}^{*}\right\}$ and $\lambda=$ $\left\langle T x_{n+1}, x_{n+1}\right\rangle$. Suppose that, for every $I>E \geq E_{m(n+1)}$ and $y \in$ $\left(E-E_{m(n+1)}\right) \tilde{H}_{n}, T y-\lambda y$ is in $\tilde{H}_{n}$. It would immediately follow that $(T-\lambda)\left(I-E_{m(n+1)}\right) \in \mathscr{F}$. That is, $T=\lambda I+F$ for some $F$ in $\mathscr{F}$, contrary to our assumption. Thus, for some $j(n+1)>m(n+1)$, we have both $y_{n+1} \in\left(E_{j(n+1)}-E_{m(n+1)}\right) H$ and $T y_{n+1}-\lambda y_{n+1} \notin \tilde{H}_{n}$.

Let $A$ be the operator

$$
A=\sum_{n=1}^{\infty} x_{n} \otimes y_{n}
$$


Now each $x_{n} \otimes y_{n}$ is in $\mathscr{A}$ and $\mathscr{A}$ is strongly closed; therefore, $A \in \mathscr{A}$. Consider the vector $w_{k}=(T A-A T) x_{k}=T y_{k}-\left\langle T x_{k}, x_{k}\right\rangle y_{k}$. From (iii) it follows that, for each $n,\left\{w_{k}\right\}_{k=1}^{n}$ are linearly independent vectors in the range of $\delta_{T}(A)$.

Case (iii). If $X$ derives $\mathscr{A}$ into $\mathscr{J}$, then $X^{*}$ derives $\mathscr{A}^{*}$ into $\mathscr{J}$. Since $\mathscr{A}^{*}=\operatorname{Alg}\left\{I-E_{n}\right\}$, where $\left\{I-E_{n}\right\}$ satisfies the hypotheses of case (ii), we obtain case (iii).

In order to prove IV, we simply combine III with the main result of [4], which says that any derivation of a nest algebra into $\mathscr{L}(H)$ is inner.

COROLLARY. It easily follows that for any generalized commutator pair $A B$, with $A T-T B$ in $\mathscr{J}$ for all $T$ in $\mathscr{A}$ implies $A, B$ are both in $C I+\mathscr{J}$.

REMARK. There has been considerable recent interest in automorphisms of perturbed algebras [14], determining under which circumstances an automorphism of $\mathscr{A}+\mathscr{J}$ is inner. For nests indexed by $\mathbf{N}$ and $\mathscr{J}=\mathscr{K}$, it is shown in [14] that every automorphism is inner. In the general situation there will exist outer automorphisms (for example, the bilateral shift acting on the classical nest of one-dimensional jumps indexed by $\mathbf{Z}$ ). Indeed, it is shown in [16] and [6] that these have a rather rich structure being isomorphic to the group of all dimension preserving order isomorphisms of the underlying nest. However, a key to all these results is the fact [2] that $\mathscr{A}+\mathscr{K}$ is precisely all operators $T$ in $\mathscr{L}(H)$ such that $E \rightarrow(I-E) T E$ is continuous from $\mathscr{E}$ (strong operator topology) to $\mathscr{K}$ (norm topology). In the situation of arbitrary (two sided) ideals, this does not hold even for tractable classes such as symmetrically normed ideals [12].

\section{REFERENCES}

[1] Dz. E. Allahverdiev, On the rate of approximation of completely continuous operators by finite dimensional operators, Azerbaidzan. Gos. Univ. Učen. Zap. Ser. Fiz.-Mat. i Him. Nauk, 2 (1957), 27-35 (Russian).

[2] W. B. Arveson, Interpolation problems in nest algebras, J. Funct. Anal., 20 (1975), 208-233.

[3] J. W. Calkin, Two-sided ideals and congruences in the ring of bounded operators on Hilbert space, Ann. Math., 42 (1941), 839-873.

[4] E. Christensen, Derivations of nest algebras, Math. Ann., 229 (1977), 155-161.

[5] E. Christensen and C. Peligrad, Commutants of nest algebras modulo the compact operators, Kobenhavns Univ. Mat. Inst. Preprint Series \# 31, Nov. 1978. 
[6] K. R. Davidson and B. H. Wagner, Automorphisms of quasitriangular algebras, preprint.

[7] J. A. Erdos, Non-selfadjoint operator algebras, Proc. R. Irish Acad. 81A (1981), 127-145.

[8] J. A. Erdos and S. Giotopoulos, On some commutators of operators, J. Operator Theory, 12 (1984), 47-64.

[9] J. A. Erdos and S. C. Power, Weakly closed ideals of nest algebras, J. Operator Theory, 7 (1982), 219-235.

[10] P. R. Halmos, A Hilbert Space Problem Book, D. van Nostrand-Reinhold, Princeton, New Jersey, 1967.

[11] B. E. Johnson and S. K. Parrott, Operators commuting with a von Neumann algebra modulo the set of compact operators, J. Funct. Anal., 11 (1972), 39-61.

[12] G. J. Knowles, $C_{p}$-perturbations of nest algebras, Proc. Amer. Math. Soc., 92 (1984), 37-40.

[13] E. C. Lance, Cohomology and perturbations of nest algebras, Proc. London Math. Soc., 43 (1981), 334-356.

[14] J. K. Plastiras, Quasitriangular operator algebras, Pacific J. Math., 64 (1976), 543-550.

[15] C. E. Rickart, General Theory of Banach Algebras, D. van Nostrand Co., Inc. (1966).

[16] B. E. Wagner, Derviations of quasitriangular algebras, Pacific J. Math., 114 (1984), 243-255.

Received August 20, 1984 and in revised form January 15, 1986.

DePARTMENT OF EleCTRICAL ENGINEERING

TEXAS TECH UNIVERSITY

LUBBOCK, TX 79409 


\section{PACIFIC JOURNAL OF MATHEMATICS EDITORS}

\author{
V. S. VARADARAJAN \\ (Managing Editor) \\ University of California \\ Los Angeles, CA 90024 \\ Herbert Clemens \\ University of Utah \\ Salt Lake City, UT 84112 \\ R. FINN \\ Stanford University \\ Stanford, CA 94305
}

\author{
HERMANN FLASCHKA \\ University of Arizona \\ Tucson, AZ 85721 \\ RAMESh A. GANGOLli \\ University of Washington \\ Seattle, WA 98195 \\ VAUGHAN F. R. JONES \\ University of California \\ Berkeley, CA 94720 \\ ROBION KIRBY \\ University of California \\ Berkeley, CA 94720 \\ C. C. MOORE \\ University of California \\ Berkeley, CA 94720 \\ H. SAMELSON \\ Stanford University \\ Stanford, CA 94305 \\ HAROLD STARK \\ University of California, San Diego \\ La Jolla, CA 92093
}

\section{ASSOCIATE EDITORS}
R. ARENS
E. F. BECKENBACH
B. H. NEUMANN
F. WOLF
K. YOSHIDA (1906-1982)

\section{SUPPORTING INSTITUTIONS}
UNIVERSITY OF ARIZONA
UNIVERSITY OF BRITISH COLUMBIA
CALIFORNIA INSTITUTE OF TECHNOLOGY
UNIVERSITY OF CALIFORNIA
MONTANA STATE UNIVERSITY
UNIVERSITY OF NEVADA, RENO
NEW MEXICO STATE UNIVERSITY
OREGON STATE UNIVERSITY
UNIVERSITY OF OREGON UNIVERSITY OF SOUTHERN CALIFORNIA STANFORD UNIVERSITY UNIVERSITY OF HAWAII UNIVERSITY OF TOKYO UNIVERSITY OF UTAH WASHINGTON STATE UNIVERSITY UNIVERSITY OF WASHINGTON 


\section{Pacific Journal of Mathematics \\ Vol. 127, No. $1 \quad$ January, 1987}

Jacob Burbea, Boundary behavior of holomorphic functions in the ball ..... 1

Jan Dijkstra, Strong negligibility of $\sigma$-compacta does not characterize

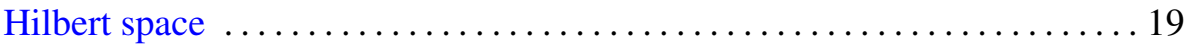

Ruy Exel, Rotation numbers for automorphisms of $C^{*}$ algebras $\ldots \ldots \ldots 31$

Howard Jacobowitz, The canonical bundle and realizable CR

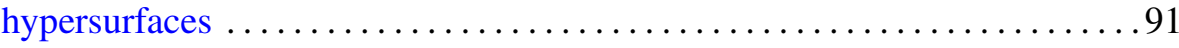

James T. Joichi and Dennis Warren Stanton, Bijective proofs of basic

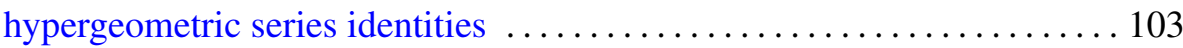

Gareth J. Knowles, Quotients of nest algebras with trivial commutator .... 121

Murray Angus Marshall, Exponentials and logarithms on Witt rings ..... 127

Courtney Hughes Moen, The dual pair $(U(3), U(1))$ over a $p$-adic field . ...141

William Ortmeyer, Surgery on a class of pretzel knots $\ldots \ldots \ldots \ldots \ldots \ldots 155$

John Gerard Ryan, Extensions of representations of Lie algebras . . . . . 173

Ivan Charles Sterling, A generalization of a theorem of Delaunay to

rotational $W$-hypersurfaces of $\sigma_{l}$-type in $H^{n+1}$ and $S^{n+1} \ldots \ldots \ldots 187$

Vesko M. Valov, Another characterization of AE(0)-spaces $\ldots \ldots \ldots \ldots \ldots 9$ 